Dermotologische Zeitschrift. 1923;38:I-IV

\title{
Contents, Vol. 38, 1923
}

Inhaltsverzeichnis.

Originalarbeiten. Seíte

Brann, Günther, Beitrag zu $\gamma$ „Linserbehandlung der

Syphilis" 333

Cohn, A., siehe Eicke.

Eicke, W., und A. Cohn, Üb $\beta \Gamma$ die neue kolloidale Liquor-

reaktion mit Benzoeharz im Vergleich mit der Goldreaktion 105 Fischl, Fr., Das

Abbauvermögen des Serums Gravider mit

Chloasma uterinum gegenüber Nebenniere 209

Gutmann, C, Zur Kenntnis der Amyloidosis der Haut . . 65 Heller, Jul., Zur Kasuistik seltener Nagelkrankheiten.

XVII. Nagelerkrankung durch Trichophyton endothrix

violaceum 13

Hirsch, Alw., Über den Einfluß des Jodkalis auf die

Syphilis, insbesondere auf die Wassermannsche Reaktion 273 Hoffmann, Erich, und R.

Strempel, Über einen Fall

von Psoriasis mit ausgedehntem großfleckigen Leukoderma

psoriaticum bei einem Säugling

Jordan, A., Schleimhautveränderungen bei Pityriasis rubra

pilaris 257

Lohmann, W., Beitrag zur Goldsolreaktion im syphilitischen

Liquor cerebrospinalis

Löwenfeld, W., Studie über den Verlauf der Lues an der

Hand gegen Therapie refraktärer Fälle 16

Mayer, Heinr., Das Krankheitsbild des Skopolenderbisses

und Skorpionstiches mit Berücksichtigung von Kriegs-

erfahrungen in Mazedonien 1

Martin, H, siehe Nathan, E.

Mühle, K., Über Bismogenol zur Luesbehandlung .... 283

Nathan, E., und H. Martin, Über Besonderheiten und

Verfeinerungsmethoden der Serodiagnostik im Primär.

stadium der Syphilis und ihre klinische Bedeutung . .321 Pakheiser, Th., Beitrag zum

Rongalitweißbild der Haut . 263 Polland, R., Dermatosis dysmenorrhoica symmetrica - ein

selbständiger Krankheitstypus 89

--, Exfoliierende Erythrodermien im Kindesalter.... 201 Strempel, Rud., siehe Hoffmann, Erich.

XY Inhaltsverzeichnis.

Seite Taehau, Paul, Bemerkungen zu der Arbeit von C. Gut-

mann: Erfahrungen über Ikterus bei Syphilitikern. . . 118

Wertheim, Leo, Über Lichtwirkung und Leukoderma bei 
Pityriasis versicolor 343

Gesellschaftsberichte.

Berliner Dermatologische Gesellschaft. Sitzung vom 9. V. 1922. 29

„20. VII. 1922. 119

»25. VII. 1922. 215

„14. XI. 1922. 286

Kölner Dermatologische Gesellschaft. Sitzungvom 27. X. 1922. 355

87. Versammlung Deutscher Naturforscher und Ärzte. Hundert-

jahrfeier: Leipzig, 17.-24. Sept. 1922. Abteilung 26:

Dermatologie und Syphilidologie. Bericht von Dr. H.

Soltmann in Leipzig - . . 126

Versammlung südwestdeutscher Dermatologen am 14. und

15. X. 1922 in Frankfurt a. M 294

Dänische Dermatologische Gesellschaft. Sitzung vom 2. III.,

6. IV., 4. V. und 5. X. 1921223

Kiewer Gesellschaft für Haut- und Geschlechtskrankheiten.

Sitzung vom 28. IX., 29. X. und 26. XI. 1922 .... 357

Periodische Liíeratur. Zusammengestellt von Dr. Arthur

Alexander in Charlottenburg . . 42, 154, 226, 298, 359

Buchbesprechungen 62, 186, 250, 317

Personalien und Tagesnachrichten. ... 63, 191, 255, 318, 371 Zum Münchener

Dermatologenkongreß (Pfingsten 1923). . . 319

Sachregister .- $\quad 372$

Namenregister 377 